\title{
Communication
}

\section{Singularities in Inflationary Cosmological Models}

\author{
Leonardo Fernández-Jambrina
}

check for updates

Citation: Fernández-Jambrina, L. Singularities in Inflationary Cosmological Models. Universe 2021, 7, 491. https://doi.org/10.3390/ universe7120491

Academic Editor: Włodzimierz Piechocki

Received: 28 October 2021

Accepted: 7 December 2021

Published: 13 December 2021

Publisher's Note: MDPI stays neutral with regard to jurisdictional claims in published maps and institutional affiliations.

Copyright: (C) 2021 by the author Licensee MDPI, Basel, Switzerland. This article is an open access article distributed under the terms and conditions of the Creative Commons Attribution (CC BY) license (https:// creativecommons.org/licenses/by/ $4.0 /)$
Matemática e Informática Aplicadas, E.T.S.I. Navales, Universidad Politécnica de Madrid, Avenida de la Memoria 4, E-28040 Madrid, Spain; leonardo.fernandez@upm.es

\begin{abstract}
Due to the accelerated expansion of the universe, the possibilities for the formation of singularities has changed from the classical Big Bang and Big Crunch singularities to include a number of new scenarios. In recent papers it has been shown that such singularities may appear in inflationary cosmological models with a fractional power scalar field potential. In this paper we enlarge the analysis of singularities in scalar field cosmological models by the use of generalised power expansions of their Hubble scalars and their scalar fields in order to describe all possible models leading to a singularity, finding other possible cases. Unless a negative scalar field potential is considered, all singularities are weak and of type IV.
\end{abstract}

Keywords: singularity; cosmology; inflation

\section{Introduction}

The formation of singularities is one of the major challenges for general relativity and has lead to plenty of conjectures and results [1] in the field of mathematical relativity. Even the term singularity comprises many different definitions, such as curvature singularities, geodesic incompleteness and b-incompleteness. Concerning cosmological scenarios, until the end of the 20th century, the only possibilities of formation of singularities were the initial Big Bang singularity and, in the case of spatially closed cosmological models, the final Big Crunch singularity, since energy conditions were enforced in these cosmological models.

However, the discovery that our universe is undergoing accelerated expansion, even presently [2-8], triggered the search for either new ingredients for the energy content of the universe (dark energy) $[9,10]$ or for modifications of the general theory of gravity that could cope with such a feature [11-13].

Since these modifications of the standard cosmological model have usually involved violation of at least one energy condition, the main theorems on singularities were not applicable and new types of singular behavior were added to the list, which was enlarged from the original proposal [14] several times [15-17]. An updated list may be found in $[18,19]$ in terms of the scale factor $a(t)$, the Hubble scalar $H(t)$ and the equation of state of the model:

- Type -1. "Grand bang/rip". Ref. [18] The scale factor becomes null or diverges for $w=-1$.

- $\quad$ Type 0 . "Big bang". The scale factor becomes null for $w \neq-1$.

- $\quad$ Type I. "Big rip" [20]. The scale factor diverges for $w \neq-1$.

- Type II. "Sudden singularities" [21-36], also named "quiescent singularities" [37]. The derivatives of the scale factor diverge from second derivative on. Some special cases are named "big brake" [38] and "big boost" [39].

- Type III. "Big freeze" [40] or "finite scale factor singularities". The derivatives of the scale factor diverge from first derivative on.

- $\quad$ Type IV. "Generalised sudden singularities [41]. The derivatives of the scale factor diverge from a derivative higher than second onward.

- Type V. "w-singularities" $[42,43]$. No derivatives of the scale factor diverge, but the barotropic index $w=p / \rho$ in the equation of state does [44]. 
- Type $\infty$. "Directional singularities" [45,46]. These happen at an infinite value of the coordinate time, but they can be reached in finite proper time by only some observers. In [1] they are dubbed p.p. curvature singularities (curvature singularities along a parallelly transported basis) in a more general framework.

Some of these singularities $(-1,0, \mathrm{I}, \infty)$ are strong [47-51] in the sense that they distort finite objects, whereas others are weak (II,IV,V) and cannot be considered either the beginning or the end of the universe.

On the other hand, such singular behavior has been shown not to appear just as possible initial or final stages of the universe, but also in inflationary models [52,53].

In that paper the authors consider cosmological models endowed with a scalar field $\phi$ under a potential $V(\phi)=A \phi^{n}$, with $A>0, n>0$. These models have been thoroughly studied for the integer $n$, since they provide classical examples of inflationary models for even $n$. However, if we allow $n$ to be a non-integer, a possibility that has been considered in the literature [54,55], new features appear in the form of sudden, generalised singularities of type IV:

- If $k<n<k+1$, where $k$ is a natural number, the derivatives of the Hubble scalar diverge from the $(k+2)$ th onward.

These singularities are not singularities in the scalar polynomials of the curvature, but in their derivatives and are named $C^{k}$ scalar curvature singularities in [56]. They were considered the first examples of realistic cosmological models wherein these singularities appeared.

In this paper we would like to enlarge the analysis of [56] by considering other scalar field potentials, making use of generalised power expansions of the Hubble scalar, the scalar field and the potential in the cosmic time coordinate. In Section 2 we perform the power expansion of the Hubble parameter and the scalar field and derive the singularities that may appear. Since the derivative of the Hubble parameter is to be negative, it is seen that just singularities of types II, IV ad V may appear in these models. In Section 3 we relate the expansion of the Hubble parameter and the expansion of the scalar field in order to derive the form of the scalar field potential of the models as a power expansion in the scalar field $\phi$. A final section of conclusions is included, where we show the list of scalar field models that develop singularities, which are seen to be of the weak type IV, unless a negative potential is considered, which allows type II singularities. The list of potentials leading to singularities includes and enlarges the one enclosed in [52,53].

\section{Divergences in the Hubble Parameter and the Scale Factor}

We focus on spatially flat homogeneous and isotropic spacetimes endowed with a metric

$$
d s^{2}=-d t^{2}+a^{2}(t)\left(d r^{2}+r^{2}\left(d \theta^{2}+\sin ^{2} \theta d \phi^{2}\right)\right),
$$

denoting by $a(t)$, the scale factor of the universe, using the cosmological time $t$ as the time coordinate. If we write down Einstein equations for these spacetimes, due to their symmetry, we end up with just two equations,

$$
\rho=\frac{3 \dot{a}^{2}}{a^{2}}, \quad p=-\frac{2 \ddot{a}}{a}-\frac{\dot{a}^{2}}{a^{2}},
$$

where $\rho(t)$ is the energy density and $p(t)$ is the pressure of the content of the universe. Derivatives with respect to $t$ are denoted by a dot. The Hubble parameter, a measure of the expansion of the universe, is defined as $H=\dot{a} / a$.

According to this, the equation of state for the content of the universe, $p=w \rho$, can be written in terms of a coefficient $w$,

$$
w=\frac{p}{\rho}=-\frac{1}{3}-\frac{2}{3} \frac{a \ddot{a}}{\dot{a}^{2}},
$$


which has been dubbed the barotropic index, which is closely related to the deceleration parameter $q$,

$$
q=-\frac{a \ddot{a}}{\dot{a}^{2}}=\frac{1+3 w}{2}
$$

and the rest of cosmographical parameters can be obtained in a similar fashion [57].

In the case of a scalar field $\phi$ subject to a potential $V(\phi)$, energy density splits into a kinetic energy and a potential energy term,

$$
\rho=\frac{\dot{\phi}^{2}}{2}+V(\phi),
$$

and so does pressure,

$$
p=\frac{\dot{\phi}^{2}}{2}-V(\phi)
$$

The Friedman equations for this configuration are then [58]

$$
3 H^{2}=\frac{\dot{\phi}^{2}}{2}+V(\phi), \quad \dot{H}=-\frac{\dot{\phi}^{2}}{2} \text {. }
$$

Deriving the first equation and replacing $\dot{H}$ with the help of the second equation, we get the evolution equation for the scalar field,

$$
\ddot{\phi}+3 H \dot{\phi}+V^{\prime}(\phi)=0 .
$$

We expect a slow-rolling scenario, for which the potential $V(\phi)$ dominates over the kinetic term $\dot{\phi}^{2}$ in the first Friedman equation. If this is true, it requires a positive scalar field potential.

The use of generalised power expansions in cosmological time has been shown useful for analysing features such as singularities in cosmology [59]. Following this idea, we assume a generalised power expansion for the Hubble parameter $H(t)$ around a time $t_{0}$,

$$
H(t)=h_{0}\left(t_{0}-t\right)^{\xi_{0}}+h_{1}\left(t_{0}-t\right)^{\xi_{1}}+h_{2}\left(t_{0}-t\right)^{\xi_{2}}+\cdots,
$$

with $\xi_{0}<\xi_{1}<\cdots$. In order to have expansion, we require $h_{0}>0$.

Since, according to the second Friedman Equation (7), the derivative of the Hubble parameter,

$$
\dot{H}(t)=-\xi_{0} h_{0}\left(t_{0}-t\right)^{\xi_{0}-1}-\xi_{1} h_{1}\left(t_{0}-t\right)^{\xi_{1}-1}-\xi_{2} h_{2}\left(t_{0}-t\right)^{\xi_{2}-1}-\cdots,
$$

must be negative in order to allow a scalar field interpretation, the product $\xi_{0} h_{0}$ must be positive. This restricts us to non-negative values for all the exponents $\xi_{i}$; and, if $\xi_{0}=0$, then $\xi_{1} h_{1}$ and hence $h_{1}$ must be positive.

We can relate $H(t)=\dot{a}(t) / a(t)$ to the scale factor $a(t)$ in order to check for singularities at $t_{0}$. Since

$$
\begin{gathered}
\ln a(t)=\ln a_{0}-\frac{h_{0}}{\xi_{0}+1}\left(t_{0}-t\right)^{\xi_{0}+1}-\frac{h_{1}}{\xi_{1}+1}\left(t_{0}-t\right)^{\xi_{1}+1}-\cdots \\
a(t)=a_{0} e^{-\frac{h_{0}}{\xi_{0}+1}\left(t_{0}-t\right)^{\xi_{0}+1}-\frac{h_{1}}{\xi_{1}+1}\left(t_{0}-t\right)^{\xi_{1}+1}-\cdots},
\end{gathered}
$$

in terms of an integration constant $a_{0}=a\left(t_{0}\right)$.

This leads to several possibilities:

- $\quad \xi_{0}=0$ : We require positive $h_{0}$ and $h_{1}$. We have finite $a\left(t_{0}\right)$ and $H\left(t_{0}\right)$ and we have type II $\left(\xi_{1}<1\right)$ or IV $\left(\xi_{1} \geq 1\right)$ singularities at $t_{0}$.

- $\quad \xi_{0}>0$ : The exponent $\xi_{0}+1$ and $h_{0}$ are positive. The scale factor $a\left(t_{0}\right)$ is finite and $H\left(t_{0}\right)$ vanishes. Depending on the value of $\xi_{0}$ we have singularities at $t_{0}$ of type II 
$\left(\xi_{0}<1\right)$ or of type IV $\left(\xi_{0} \geq 1\right)$ with a vanishing Hubble factor. If $\xi_{0} \geq 2$ and every $\xi_{i}$ is natural, we would have a type $\mathrm{V}$ singularity.

\section{Reconstruction of the Scalar Field Potential}

We may reconstruct the scalar field potential $V(\phi)$ from the Friedman equations.

According to the second Friedman Equation (7),

$$
\dot{\phi}(t)= \pm \sqrt{-2 \dot{H}(t)}= \pm \sqrt{2 h_{0} \xi_{0}}\left(t_{0}-t\right)^{\left(\tilde{\xi}_{0}-1\right) / 2}+\cdots
$$

the scalar field behaves as

$$
\phi(t)=\phi_{0} \mp \frac{2 \sqrt{2 h_{0} \tau_{0}}}{\xi_{0}+1}\left(t_{0}-t\right)^{\left(\xi_{0}+1\right) / 2}+\cdots,
$$

in terms of an integration constant $\phi_{0}$, whereas we get the time evolution of the potential from the first Friedman equation,

$$
\begin{aligned}
V(\phi(t)) & =3 H^{2}(t)+\dot{H}(t)=3 h_{0}^{2}\left(t_{0}-t\right)^{2 \xi_{0}}+6 h_{0} h_{1}\left(t_{0}-t\right)^{\xi_{0}+\xi_{1}}+3 h_{1}^{2}\left(t_{0}-t\right)^{2 \xi_{1}} \\
& +6 h_{0} h_{2}\left(t_{0}-t\right)^{\xi_{0}+\xi_{2}}+6 h_{1} h_{2}\left(t_{0}-t\right)^{\xi_{1}+\xi_{2}}+3 h_{2}^{2}\left(t_{0}-t\right)^{2 \xi_{2}}+\cdots \\
& -\xi_{0} h_{0}\left(t_{0}-t\right)^{\xi_{0}-1}-\xi_{1} h_{1}\left(t_{0}-t\right)^{\xi_{1}-1}-\xi_{2} h_{2}\left(t_{0}-t\right)^{\xi_{2}-1}-\cdots .
\end{aligned}
$$

For $\xi_{0}<-1$, the lowest order corresponds to the $2 \xi_{0}$ term, whereas for $\xi_{0}>-1$ it is the $\xi_{0}-1$ one unless $\xi_{0}=0$, but we have already excluded models with negative $\xi_{0}$. However, negative $\xi_{0}$ has been considered for intermediate inflation models [60].

Additionally, according to the slow roll approximation, the $2 \xi_{0}$ term is expected to dominate over the $\xi_{0}-1$ term. This would happen only for models with non-positive $\xi_{0}$. Hence the only inflationary models with positive potential are those with $\xi_{0}=0$. Inflationary models with $\xi_{0}>0$ would have a negative scalar field potential.

Let us consider these cases:

- $\quad$ Non-natural $\xi_{0}>0: V(\phi(t)) \simeq-\xi_{0} h_{0}\left(t_{0}-t\right)^{\xi_{0}-1} \propto\left(\phi(t)-\phi_{0}\right)^{\eta} \propto\left(t_{0}-t\right)^{\left(\xi_{0}+1\right) \eta / 2}$, with $\eta=2\left(\xi_{0}-1\right) /\left(\xi_{0}+1\right)$. This means a leading power $\eta \in(-2,2)$ in the negative potential and vanishing $H\left(t_{0}\right)$. For $\eta \in(-2,0)$ we have a type II singularity. Otherwise we have a type IV singularity.

- $\xi_{0}=0$ : This is the case of a finite $H\left(t_{0}\right)$,

$$
\begin{gathered}
H(t)=h_{0}+h_{1}\left(t_{0}-t\right)^{\xi_{1}}+\cdots, \\
\dot{H}(t)=-\xi_{1} h_{1}\left(t_{0}-t\right)^{\xi_{1}-1}+\cdots, \\
\dot{\phi}(t) \simeq \pm \sqrt{2 h_{1} \xi_{1}}\left(t_{0}-t\right)^{\left(\xi_{1}-1\right) / 2},
\end{gathered}
$$

where $\xi_{1}, h_{1}>0$. The expansions of the scalar field and the time evolution of the potential are, in this case,

$$
\begin{aligned}
& \phi(t)=\phi_{0} \mp \frac{2 \sqrt{2 h_{1} \xi_{1}}}{\xi_{1}+1}\left(t_{0}-t\right)^{\left(\xi_{1}+1\right) / 2}+\cdots, \\
V(\phi(t))= & 3 h_{0}^{2}+6 h_{0} h_{1}\left(t_{0}-t\right)^{\xi_{1}}+3 h_{1}^{2}\left(t_{0}-t\right)^{2 \xi_{1}} \\
+ & 6 h_{0} h_{2}\left(t_{0}-t\right)^{\xi_{2}}+6 h_{1} h_{2}\left(t_{0}-t\right)^{\xi_{1}+\xi_{2}}+3 h_{2}^{2}\left(t_{0}-t\right)^{2 \xi_{2}}+\cdots \\
- & \xi_{1} h_{1}\left(t_{0}-t\right)^{\xi_{1}-1}-\xi_{2} h_{2}\left(t_{0}-t\right)^{\xi_{2}-1}-\cdots,
\end{aligned}
$$

leading to several interesting subcases: 
- $\xi_{1}<1: V(\phi(t)) \simeq-\xi_{1} h_{1}\left(t_{0}-t\right)^{\xi_{1}-1} \propto\left(\phi(t)-\phi_{0}\right)^{2\left(\xi_{1}-1\right) /\left(\xi_{1}+1\right)}$, and $V(\phi)$ behaves as a power $\left(\phi-\phi_{0}\right)^{\eta}$ with $\eta \in(-2,0)$. For such models $H\left(t_{0}\right)$ is finite, but not $\dot{H}\left(t_{0}\right)$. That is, a type II singularity, but with a negative potential.

The rest of the subcases correspond to type IV singularities and positive scalar field potentials:

- Non-natural $\xi_{1}>1: V(\phi(t)) \simeq 3 h_{0}^{2}-\xi_{1} h_{1}\left(t_{0}-t\right)^{\xi_{1}-1} \simeq 3 h_{0}^{2}-\alpha \xi_{1} h_{1}$ $\left(\phi(t)-\phi_{0}\right)^{2\left(\xi_{1}-1\right) /\left(\xi_{1}+1\right)}$, and the potential behaves as a positive constant plus a term $\left(\phi-\phi_{0}\right)^{\eta}$, with exponent $\eta=2\left(\xi_{1}-1\right) /\left(\xi_{1}+1\right)$ in the interval $(0,2) . \dot{H}\left(t_{0}\right)$ vanishes and $H^{(n)}\left(t_{0}\right)$ diverges for $\eta \in\left(\frac{2(n-2)}{n}, \frac{2(n-1)}{n+1}\right), n \geq 2$.

- $\xi_{1}=1$ : Type IV singularities with finite $\dot{H}\left(t_{0}\right)$ may arise in this case:

$$
V(\phi(t))=3 h_{0}^{2}-h_{1}+6 h_{0} h_{1}\left(t_{0}-t\right)+3 h_{1}^{2}\left(t_{0}-t\right)^{2}-\xi_{2} h_{2}\left(t_{0}-t\right)^{\xi_{2}-1} \cdots,
$$

and a leading linear evolution for the scalar field,

$$
\phi(t)=\phi_{0} \mp \sqrt{2 h_{1}}\left(t_{0}-t\right)+\cdots .
$$

Again, several possibilities arise:

- $h_{1} \neq 3 h_{0}^{2}$ :

For $\xi_{2} \in(1,2)$, the leading non-constant term in $V(\phi(t))$ is the one with exponent $\xi_{2}-1$. The potential behaves as a constant plus a term $\left(\phi-\phi_{0}\right)^{\eta}$, with $\eta=\xi_{2}-1 \in(0,1)$. The singularity appears for $\ddot{H}\left(t_{0}\right)$.

For $\xi_{2}>2$ or $\xi_{2}=2, h_{2} \neq 3 h_{0} h_{1}$, the leading non-constant term in $V(\phi(t))$ is the linear one. The potential behaves as a constant plus a linear term in $\phi$. The singularity would appear for $\dddot{H}\left(t_{0}\right)$.

We may produce scalar field potentials with a higher leading power, besides the constant term, by requiring some cancellations between terms. We reproduce here the cases of quadratic, cubic and quartic powers:

For $\xi_{2}=2, h_{2}=3 h_{0} h_{1}$,

$$
V(\phi(t))=3 h_{0}^{2}-h_{1}+\left(18 h_{0}^{2}+3 h_{1}\right) h_{1}\left(t_{0}-t\right)^{2}-\xi_{3} h_{3}\left(t_{0}-t\right)^{\xi_{3}-1}+\cdots,
$$

and the potential behaves as a constant plus a quadratic term in $\phi$.

- $h_{1}=-6 h_{0}^{2}, \xi_{2}=2, h_{2}=-18 h_{0}^{3}$ :

$$
V(\phi(t))=9 h_{0}^{2}-\xi_{3} h_{3}\left(t_{0}-t\right)^{\xi_{3}-1}+648\left(t_{0}-t\right)^{3}+\cdots,
$$

For $\xi_{3} \in(2,4)$, the potential behaves as a constant plus a term $\phi^{\eta}$, with $\eta=$ $\xi_{3}-1 \in(1,3)$. The singularity appears for $\dddot{H}\left(t_{0}\right)$ if $\eta \in(1,2)$ and for $\dddot{H}\left(t_{0}\right)$ if $\eta \in(2,3)$.

For $\xi_{3}>4$, the potential behaves as a constant plus a cubic term in $\phi$.

For $\xi_{3}=4, h_{3}=162 h_{0}^{5}, \xi_{4} \in(4,5)$,

$$
V(\phi(t))=9 h_{0}^{2}-\xi_{4} h_{4}\left(t_{0}-t\right)^{\xi_{4}-1}+1944 h_{0}^{6}\left(t_{0}-t\right)^{4}+\cdots,
$$

the potential behaves as a constant plus a term $\left(\phi-\phi_{0}\right)^{\eta}$, with $\eta=\xi_{4}-1 \in(3,4)$. $\dddot{H}\left(t_{0}\right)$ is regular now.

For $\xi_{3}=4, h_{3}=162 h_{0}^{5}, \xi_{4}>5$ :

$$
V(\phi(t))=9 h_{0}^{2}-\xi_{4} h_{4}\left(t_{0}-t\right)^{\xi_{4}-1}+1944 h_{0}^{6}\left(t_{0}-t\right)^{4}+\cdots,
$$

and the potential behaves as a constant plus a quartic term in $\phi$.

We see clearly that they follow the singularity pattern in $[52,53]$ for the derivatives of the Hubble parameter, despite the additional terms. 
We may further remove the constant term in the scalar field potential to recover the cases in [52,53]. We produce some cases in order to show how the singular derivatives of the Hubble parameter come up:

- $\quad h_{1}=3 h_{0}^{2}$ :

$$
V(\phi(t))=18 h_{0}^{3}\left(t_{0}-t\right)+27 h_{0}^{4}\left(t_{0}-t\right)^{2}-\xi_{2} h_{2}\left(t_{0}-t\right)^{\xi_{2}-1} \cdots
$$

For $\xi_{2} \in(1,2)$, the potential goes as $\left(\phi-\phi_{0}\right)^{\eta}$ with $\eta=\xi_{2}-1 \in(0,1)$ and the singularity appears for $\ddot{H}\left(t_{0}\right)$.

For $\xi_{2}>2$, the potential is linear in $\phi$. And the same happens for $\xi_{2}=2, h_{2} \neq 9 h_{0}^{3}$. $\ddot{H}\left(t_{0}\right)$ is regular now.

For $\xi_{2}=2, h_{2}=9 h_{0}^{3}$,

$$
V(\phi(t))=81 h_{0}^{4}\left(t_{0}-t\right)^{2}+162 h_{0}^{5}\left(t_{0}-t\right)^{3}-\xi_{3} h_{3}\left(t_{0}-t\right)^{\xi_{3}-1}+\cdots,
$$

we have for $\xi_{3} \in(2,3)$ a potential which goes as $\left(\phi-\phi_{0}\right)^{\eta}$ with $\eta=\xi_{3}-1 \in(1,2)$ and a quadratic potential for $\xi_{3}>3$ or $\xi_{3}=3, h_{3} \neq 27 h_{0}^{4}$. $\dddot{H}\left(t_{0}\right)$ is regular in the latter case.

For $\xi_{2}=2, h_{2}=9 h_{0}^{3}, \xi_{3}=3, h_{3}=27 h_{0}^{4}$,

$$
V(\phi(t))=324 h_{0}^{5}\left(t_{0}-t\right)^{3}+729 h_{0}^{6}\left(t_{0}-t\right)^{4}-\xi_{4} h_{4}\left(t_{0}-t\right)^{\xi_{4}-1}+\cdots,
$$

we have for $\xi_{4} \in(3,4)$ a potential which goes as $\left(\phi-\phi_{0}\right)^{\eta}$ with $\eta=\xi_{4}-1 \in(2,3)$ and a cubic potential for $\xi_{4}>4$ or $\xi_{4}=4, h_{4} \neq 81 h_{0}^{5}$. $\dddot{H}\left(t_{0}\right)$ is regular in the latter case.

\section{Conclusions}

In the previous section we have identified the scalar field potentials that lead to singularities. We may collect them from the cases that have arisen.

For scalar field potentials of the form, $\eta_{0}<\eta_{1}<\cdots$,

$$
V(\phi)=V_{0}\left(\phi-\phi_{0}\right)^{\eta_{0}}+V_{1}\left(\phi-\phi_{0}\right)^{\eta_{1}}+\cdots \cdots,
$$

we have obtained type IV singularities in higher derivatives of the Hubble parameter:

- $\quad V(\phi)=V_{0}+\cdots+V_{n-2}\left(\phi-\phi_{0}\right)^{n-2}+V_{\eta}\left(\phi-\phi_{0}\right)^{\eta}+\cdots, \eta \in(n-2, n-1), n=$ $2,3, \ldots$, and finite $\dot{H}\left(t_{0}\right)$ : The first singular derivative of the Hubble parameter is $H^{(n)}\left(t_{0}\right)$. These include the cases studied in the paper by Barrow and Graham $[52,53]$.

- $\quad V(\phi)=V_{0}+V_{\eta}\left(\phi-\phi_{0}\right)^{\eta}+\cdots, \eta \in(0,2)$ and vanishing $\dot{H}\left(t_{0}\right): H^{(n)}\left(t_{0}\right)$ is singular for $\eta \in\left(\frac{2(n-2)}{n}, \frac{2(n-1)}{n+1}\right), n \geq 2$.

Additionally, if we allow the scalar field potential to be negative:

- $\quad \eta_{0} \in(-2,0)$ : Type II singularity.

- $\quad \eta_{0} \in[0,2)$ and vanishing $H\left(t_{0}\right)$ : Type IV singularity.

Summarizing, we have performed a thorough analysis of the singularities that may arise in scalar field cosmologies of the form (7) and obtained just Type IV singularities, except in the case of a negative potential, for which Type II singularities may appear.

These results extend, and of course comprise, those in [52,53] for fractional power scalar field potentials. Aside from the case of negative scalar field potentials and the extension of the form of the potential with additional terms in natural powers of $\phi$, we see that, on considering a vanishing value of $\dot{H}\left(t_{0}\right)$, singularities appear for potentials of the form $V(\phi)=V_{0}+V_{\eta}\left(\phi-\phi_{0}\right)^{\eta}+\cdots, \eta \in(0,2)$. Such singularities may appear on higher derivatives of the Hubble parameter, starting at $H^{(n)}\left(t_{0}\right)$, for $n$ as large as required.

These singularities are weak $[48,49,51]$ and in this sense they do not imply the end of the universe, since finite objects may cross them without disruption and spacetime can be extended beyond the singularity, which may be interpreted in terms of shock waves [61] 
propagating through spacetime. Energy conditions are not violated and energy density and pressure remain finite and only higher derivatives diverge.

Funding: This research received no external funding

Informed Consent Statement: Not applicable.

Data Availability Statement: Not applicable.

Acknowledgments: The author wishes to thank the University of the Basque Country for their hospitality.

Conflicts of Interest: The author declares no conflict of interest.

\section{References}

1. Hawking, S.W.; Ellis, G.F.R. The Large Scale Structure of Space-Time; Cambridge University Press: Cambridge, UK, 1973.

2. Riess1, A.G; Filippenko, A.V.; Challis, P; Clocchiatti, A.; Diercks, A; Garnavich, P.M; Gilliland, R.L.; Hogan, C.J; Jha, S; Kirshner, R.P.; et al. Observational Evidence from Supernovae for an Accelerating Universe and a Cosmological Constant. Astron. J. 1998, $116,1009$.

3. Perlmutter, S.; Aldering, G.; Goldhaber, G.; Knop, R.A.; Nugent, P.; Castro, P.G.; Deustua, S.; Fabbro, S.; Goobar, A.; Groom, D.E.; et al. Measurements of $\Omega$ and $\Lambda$ from 42 High-Redshift Supernovae. Astrophys. J. 1999, 517, 565. [CrossRef]

4. Davis, T.M. ; Mörtsell, E.; Sollerman, J; Becker, A.C.; Blondin, S.; Challis, P.; Clocchiatti, A.; Filippenko, A.V.; Foley, R.J.; Garnavich, P.M.; et al. Scrutinizing exotic cosmological models using ESSENCE supernova data combined with other cosmological probes. Astrophys. J. 2007, 666, 716. [CrossRef]

5. Wood-Vasey, W.M.; Miknaitis, G.; Stubbs, C.W.; Jha, S.; Riess, A.G.; Garnavich, P.M.; Kirshner, R.P.; Aguilera, C.; Becker, A.C.; Blackman, J.W.; et al. Observational Constraints on the Nature of the Dark Energy: First Cosmological Results from the ESSENCE Supernova Survey. Astrophys. J. 2007, 666, 694. [CrossRef]

6. Leibundgut, B. Cosmology with Supernovae. Reviews of Modern Astronomy; Schielicke, R.E., Ed.; Wiley-VCH: Weinheim, Germany, 2004; Volume 17.

7. $\quad$ Spergel, D.N.; Verde, L.; Peiris1, H.V; Komatsu, E.; Nolta, M.R.; Bennett, C.L.; Halpern, M.; Hinshaw, G.; Jarosik, N.; Kogut A.; et al. First Year Wilkinson Microwave Anisotropy Probe (WMAP) Observations: Determination of Cosmological Parameters. Astrophys. J. Suppl. 2003, 148, 175. [CrossRef]

8. Spergel, D.N.; Bean, R.; Doré, O.; Nolta, M.R.; Bennett, C.L.; Dunkley, J.; Hinshaw, G.; Jarosik, N.; Komatsu, E.; Page, L.; et al. Wilkinson Microwave Anisotropy Probe (WMAP) three year results: Implications for cosmology. Astrophys. J. Suppl. 2007, 170, 377. [CrossRef]

9. Padmanabhan, T. Dark Energy: Mystery of the Millennium. AIP Conference Proceedings 2006, 861, 179.

10. Sahni, V.; Starobinsky, A. Reconstructing dark energy. Int. J. Mod. Phys. D 2006, 15, 2105. [CrossRef]

11. Maartens, R. Dark Energy and Dark Gravity. J. Phys. Conf. Ser. 2007, 68, 012046. [CrossRef]

12. Durrer, R.; Maartens, R. Dark energy and dark gravity: theory overview. Gen. Rel. Grav. 2008, 40, 301. [CrossRef]

13. Fernández-Jambrina, L.; Lazkoz, R. Singular fate of the universe in modified theories of gravity. Phys. Lett. B 2009, 670, 254. [CrossRef]

14. Nojiri, S.; Odintsov, S.D.; Tsujikawa, S. Properties of singularities in (phantom) dark energy universe. Phys. Rev. D 2005, 71, 063004. [CrossRef]

15. Da̧browski, M.P.D.; Marosek, K. Regularizing cosmological singularities by varying physical constants. JCAP $20132,12$. [CrossRef]

16. Yurov, A.V. Brane-like singularities with no brane. Phys. Lett. B 2010, 689, 1. [CrossRef]

17. Dąbrowski, M.P.; Marosek, K.; Balcerzak, A. Standard and exotic singularities regularized by varying constants. Mem. Della Soc. Astron. 2014, 85, 44.

18. Fernández-Jambrina, L. Grand rip and grand bang/crunch cosmological singularities. Phys. Rev. D 2014, 90, 064014. [CrossRef]

19. Lazkoz, R.; Fernández-Jambrina, L. Singularities in inflationary cosmological models. arXiv, 2021, arXiv:2112.03647.

20. Caldwell, R.R.; Kamionkowski, M.; Weinberg, N.N. Phantom Energy and Cosmic Doomsday. Phys. Rev. Lett. $2003,91071301$. [CrossRef]

21. Barrow, J.D. Sudden future singularities. Class. Quant. Grav. 2004, 21, L79. [CrossRef]

22. Nojiri, S.; Odintsov, S.D. Quantum escape of sudden future singularity. Phys. Lett. B 2004, 595, 1. [CrossRef]

23. Barrow, J.D. More general sudden singularities. Class. Quant. Grav. 2004, 21, 5619. [CrossRef]

24. Lake, K. Sudden future singularities in FLRW cosmologies. Class. Quant. Grav. 2004, 21, L129. [CrossRef]

25. Nojiri, S.; Odintsov, S.D. Final state and thermodynamics of a dark energy universe. Phys. Rev. D 2004, 70, 103522. [CrossRef]

26. Da̧browski, M.P. Inhomogenized sudden future singularities. Phys. Rev. D 2005, 71, 103505. [CrossRef]

27. Chimento, L.P.; Lazkoz, R. On big rip singularities. Mod. Phys. Lett. A 2004, 19, 2479. [CrossRef]

28. Dabbrowski, M.P. Statefinders, higher-order energy conditions and sudden future singularities. Phys. Lett. B 2005, 625, 184. [CrossRef] 
29. Barrow, J.D.; Batista, A.B.; Fabris, J.C.; Houndjo, S. Quantum particle production at sudden singularities. Phys. Rev. D 2008, 78, 123508. [CrossRef]

30. Barrow, J.D.; Lip, S.Z.W. Classical stability of sudden and big rip singularities. Phys. Rev. D 2009, 80, 043518. [CrossRef]

31. Nojiri, S.; Odintsov, S.D. Future evolution and finite-time singularities in $\mathrm{F}(\mathrm{R})$ gravity unifying inflation and cosmic acceleration. Phys. Rev. D 2008, 78, 046006. [CrossRef]

32. Barrow, J.D.; Cotsakis, S.; Tsokaros, A. A general sudden cosmological singularity. Class. Quant. Grav. 2010, 27, 165017. [CrossRef]

33. Singh, P. Curvature invariants, geodesics, and the strength of singularities in Bianchi-I loop quantum cosmology. Phys. Rev. D 2012, 85, 104011. [CrossRef]

34. Denkiewicz, T.; Dąbrowski, M.P.; Ghodsi, H.; Hendry, M.A. Cosmological tests of sudden future singularities. Phys. Rev. D 2012, 85, 083527. [CrossRef]

35. Barrow, J.D.; Galloway, G.J.; Tipler, F.J. The closed-universe recollapse conjecture. MNRAS 1986, 223, 835. [CrossRef]

36. Fernández-Jambrina, L.; Lazkoz, R. Geodesic behaviour of sudden future singularities. Phys. Rev. D 2004, 70, 121503. [CrossRef]

37. Shtanov, Y.; Sahni, V. New cosmological singularities in braneworld models. Class. Quant. Grav. 2002, 19, L101. [CrossRef]

38. Gorini, V.; Kamenshchik, A.Y.; Moschella, U.; Pasquier, V. Tachyons, scalar fields, and cosmology. Phys. Rev. D 2004, 69, 123512. [CrossRef]

39. Barvinsky, A.O.; Deffayet, C.; Kamenshchik, A.Y. CFT driven cosmology and the DGP/CFT correspondence. JCAP 2010, 5, 34. [CrossRef]

40. Bouhmadi-López, M.; Gonzalez-Díaz, P.F.; Martín-Moruno, P. Worse than a big rip? Phys. Lett. B 2008, 659, 1. [CrossRef]

41. Barrow, J.D.; Tsagas, C.G. New Isotropic and Anisotropic Sudden Singularities. Class. Quant. Grav. 2005, 22, 1563. [CrossRef]

42. Dąbrowski, M.P.; Denkiewicz, T. Barotropic index w-singularities in cosmology. Phys. Rev. D 2009, 79, 063521. [CrossRef]

43. Shtanov, Y.; Sahni, V. Did the universe loiter at high redshifts? Phys. Rev. D 2005, 71, 084018.

44. Fernández-Jambrina, L. W-cosmological singularities. Phys. Rev. D 2010, 82, 124004. [CrossRef]

45. Fernández-Jambrina, L. Hidden past of dark energy cosmological models. Phys. Lett. B 2007, 656, 9. [CrossRef]

46. Fernández-Jambrina, L. Initial directional singularity in inflationary models. Phys. Rev. D 2016, 94, 024049. [CrossRef]

47. Ellis, G.F.R.; Schmidt, B.G. Singular space-times. Gen. Rel. Grav. 1977, 8, 915. [CrossRef]

48. Tipler, F.J. Singularities in conformally flat spacetimes. Phys. Lett. A 1977, 64, 8. [CrossRef]

49. Królak, A. Towards the proof of the cosmic censorship hypothesis. Class. Quant. Grav. 1986, 3, 267. [CrossRef]

50. Rudnicki, W.; Budzynski, R.J.; Kondracki, W. Generalized strong curvature singularities and weak cosmic censorship in cosmological space-times. Mod. Phys. Lett. A 2006, 21, 1501. [CrossRef]

51. Fernández-Jambrina, L.; Lazkoz, R. Classification of cosmological milestones. Phys. Rev. D 2006, 74, 064030. [CrossRef]

52. Barrow, J.D.; Graham, A.A.H. Singular Inflation. Phys. Rev. D 2015, 91, 083513. [CrossRef]

53. Barrow, J.D.; Graham, A.A.H. New Singularities in Unexpected Places. Int. J. Mod. Phys. D 2015 24, 1544012. [CrossRef]

54. Harigaya, K.; Ibe, M.; Schmitz, K.; Yanagida, T.T. Chaotic inflation with a fractional power-law potential in strongly coupled gauge theories. Phys. Lett. B 2013, 720, 125-129. [CrossRef]

55. Harigaya, K.; Ibe, M.; Schmitz, K.; Yanagida, T.T. Dynamical fractional chaotic inflation. Phys. Rev. D 2014, 90, 123524. [CrossRef]

56. Ellis, G.F.R.; Schmidt, B. Classification of singular space-times. Gen. Rel. Grav. 1979, 10, 989-997. [CrossRef]

57. Visser, M. Cosmography: Cosmology without the Einstein equations. Gen. Relativ. Gravit. 2005, 37, 1541-1548. [CrossRef]

58. Liddle, A.R.; Lyth, D.H. Cosmological Inflation and Large-Scale Structure; Cambridge University Press: Cambridge, UK, 2000.

59. Cattoën, C.; Visser, M. Necessary and sufficient conditions for big bangs, bounces, crunches, rips, sudden singularities, and extremality events. Class. Quant. Grav. 2005, 22, 4913. [CrossRef]

60. Barrow, J.D.; Saich, P. The behaviour of intermediate inflationary universes. Phys. Lett. B 1990, 249, 406-410. [CrossRef]

61. Barrow, J.D.; Cotsakis, S.; Trachilis, D. The generic sudden singularity in Brans-Dicke theory. Eur. Phys. J. C 2020, 80, 1197. [CrossRef] 